\title{
Employees with Asperger's Syndrome and their Experiences within the Work Environment
}

\author{
Anna Julian \\ Undergraduate \\ Department of Psychology, Dublin Business School \\ Dublin, Ireland. \\ Dr. Ronda Barron \\ Lecturer \\ Department of Psychology, Dublin Business School \\ Dublin, Ireland.
}

(C) Anna Julian and Ronda Barron. This work is licensed under the Creative Commons Attribution-NonCommercial-ShareAlike 4.0 International License. To view a copy of this license, visit https://creativecommons.org/licenses/by-nc-sa/4.0/ .

\begin{abstract}
This qualitative study gathered experiences of employees with Asperger's Syndrome (AS) within their workplaces. Data were collected by conducting six semi-structured interviews: three face-to-face, one by phone and two by Skype with audio only. A thematic analysis with an inductive approach was applied. Four main themes with multiple sub-themes emerged: Competence and Work Performance; Self-improvement and Career Progression; Supportive Work Environment; and AS in the Workplace. Findings revealed that these employees were team-oriented, productive and highly skilled professionals with a strong work ethic. Furthermore, their diagnoses did not hinder fulfilling careers. They emphasised knowledge sharing and factual communication while cooperating with their work colleagues. However, decoding workplace politics presented a major hurdle to their job satisfaction. Also, the traditional hiring process, as well as bright fluorescent overhead lights and noisy surroundings (for example canteens, office spaces) were sources of distraction and distress. Therefore, the essential role of HR would be to re-visit and adjust the interview process. Whereas managers should, perhaps, reflect on their leadership and communication styles, show some recognition for their employees' quality-consciousness and become advocates of a more inclusive culture. Finally, enabling career-progression and providing sufficient learning opportunities for these employees should also be prioritised by managers.

Keywords: Asperger's Syndrome; Workplace; Experience; Employment; Career; Inclusive Culture; Job Interview; Teamwork; Productivity; Leadership Styles.
\end{abstract}

\section{Introduction}

The dynamic nature of the current job market forces businesses to embrace diversity in the workforce. Thus, an evident increased interest in the topic of employees with a diagnosis of Asperger's Syndrome (AS) should come as no surprise. As per the Department of Health (DOH) (2018), the prevalence of Autistic Spectrum Disorder (ASD) in Ireland is estimated at 1.5\%; however, this estimation does not include the 
adult population. Moreover, the employability rate within this population in Ireland is still unknown but according to international estimates, among the approximate $1 \%$ of the diagnosed individuals, only as little as 6\% are in employment (Wareham and Sonne, 2008). Despite declarations on promoting an inclusive culture, unfortunately in some organisations employees with AS are often deeply misunderstood whereas prejudice and ignorance of AS, are still alive and well (Hendrickx, 2008).

Hans Asperger's paper on autism was published back in 1944 but only fifty years later, AS was distinguished from autism (Lyons and Fitzgerald, 2007). However, since 2013, the two were amalgamated and, as per the Diagnostic and Statistical Manual of Mental Disorders (DSM-5), classified as ASD (Skuse, 2018). It is important to note that symptoms vary in their severity, thus the word spectrum was applied in this classification. As outlined by the American Psychiatric Association (2013), ASD manifested itself by symptoms like difficulty in reading social cues, hypersensitivity to visual or auditory stimuli (bright lights, noisy surroundings etc.) or strong dislike of changes. To this day, however, these diagnoses' amalgamation is debatable and, to some health specialists, even highly controversial. The held belief being that autism and AS are too distinct to be merged and, more importantly, therapeutic interventions for each differ (Kite, Gullifer and Tyson, 2013). As emphasised by Kite, Gullifer and Tyson (2013), autism often has negative connotations, perceived by some as a more severe diagnosis; in contrast AS is often attributed to savants or high achievers. These misconceptions often lead to concealment of diagnosis by AS employees due to fear of autism stereotyping. Concealment prevents being pigeonholed as disabled but also does not hinder the opportunity to be hired or simply to retain their current employment (Johnson and Joshi, 2016; Hendrickx, 2008).

Lorenz and Heinitz (2014) took notice that AS is, unfortunately, often perceived as a disability and such stigmatisation resulted in low self-esteem within the population of employees who received an official diagnosis. However, Austin and Pisano (2017) had a different perspective on this population; rather than focusing on stigmatisation issues, they believed these employees just had a different cognitive style. Furthermore, many employees, instead of viewing their own AS as a disability, fully embraced it and actually viewed it as an advantage. As reported in the qualitative study with six participants conducted by Krieger et al. (2012), they had a sense of achievement and, despite any difficulties related to AS they might have encountered at work, many of them still managed to have successful careers. However, at times, sustaining jobs and having a continuity of employment was truly problematic to some. Despite being highly competent and having qualities which would only make them assets to any organisation, (during or after the probationary period had passed) they were evaluated by some employers as inflexible or not team-oriented (Parr, Hunter and Ligon, 2013; Scott et al., 2015).

Traditional interview processes which focus heavily on communication skills may not provide individuals with AS a platform to demonstrate their true expertise. Additional methods of assessment have been identified to provide practical assessment of candidates' skills; one Danish consulting company Specialisterne (The Specialists) replaced the traditional interview with a process called hangouts. This method is an equivalent of an assessment centre and enables practical assessment of candidates' problem-solving skills by means of the LEGO Mindstorms (Austin and Pisano, 2017). Specialisterne posit that embracing diversity by hiring candidates who may be 
perceived as outliers (i.e. they do not fit to the traditional workplace), could only benefit an organisation (Austin and Pisano, 2017).

When candidates with AS are hired, as emphasised both at the review article by Austin and Pisano (2017) and the qualitative study conducted by Scott et al. (2015), these employees could excel in their jobs, as long as their employer is willing to put some minor adjustments into their physical work environment to reduce sensory overload. This involves adjusting overhead desk lighting, providing noise-cancelling headphones or assigning a workstation facing the wall to minimise distractions (Austin and Pisano, 2017; Hedley et al., 2018). Such enablers, as defined by Hedley et al. (2018), are feasible and inexpensive yet contribute to increased productivity, overall job satisfaction and, more importantly, benefit the whole organisation. However, once an individual with AS has entered into employment, a myriad of other difficulties are often reported such as social interactions, inflexibility (dislike of unexpected changes) and sensory overload caused by a noisy over-stimulating environment (Parr and Hunter, 2014). Communication difficulties, in particular "office banter", included difficulties in interpreting facial expressions or understanding the sarcastic sense of humour of their colleagues (Vogeley et al., 2013). Conversely, work tasks and the job itself were not reported as problems (Scott et al., 2015).

In contrast with most of the previous research on this population, one AS employment support service, Specialisterne, emphasised strengths and aptitudes of employees with AS. They also believed that once these workers' talents are utilised and tailored to the specific role, this, as a result, contributed to the success of any organisation. It was reported that, for example, they could excel in quality control due to their exceptional accuracy as they notice tiny but important details that can often be overlooked by their colleagues (Wareham and Sonne, 2008). Besides, as per findings from the qualitative study with 52 employees with AS conducted by Parr, Hunter and Ligon (2013), while their team members find certain tasks tedious, they are comfortable performing them both thoroughly and efficiently. Their strong work ethic certainly did not go unnoticed. As discussed by Hedley et al. (2018), focus groups and interviews revealed that other team members admired productivity, commitment and outstanding quality of work performed by their colleagues with AS. Furthermore, as reported in the case study by Wareham and Sonne (2008), employees with AS outperformed their colleagues as they turned out to be eight times more accurate in data input work. Whereas in the field of software testing, their accuracy was $50 \%$ greater in comparison to other team members.

Despite some unfair misconceptions about these employees being supposedly unsuitable for customer service roles, Austin and Pisano (2017) described an example of an employee with AS, employed by SAP, who resolved a commonly encountered issue for the client by simply applying a different cognitive style. As a result, not only the customer was satisfied but also the whole organisation's credibility and reputation were strengthened. In fact, employees with AS provided flawless phone-based customer service as this type of interaction was more comfortable to them. Since they sometimes find eye contact intimidating, it suited them to have structured, pre-written and scripted answers which they could apply while responding to phone queries (Johnson and Joshi, 2016; Trevisan et al., 2017). 
As suggested by Parr, Hunter and Ligon (2013), the leadership style used by managers while interacting with staff members with a diagnosis of Asperger's mattered. One example of a leadership type is the transformational leadership style; in essence, transformational leaders are supportive and mindful of their employees' needs. Whereas to motivate staff, these leaders tend to use an emotion-laden communication style but according to Parr, Hunger and Ligon (2013), it was ineffective during interactions with staff members who were diagnosed with Asperger's. Parr, Hunter and Ligon (2013) noted that this communication style increased their anxiety, whereas abstract concepts, used by transformational leaders, unfortunately only amplified misunderstandings. It is because individuals diagnosed with Asperger's can sometimes misread metaphors as they take them literally. Moreover, it was reported that they prefer directness and honest feedback from their managers. They thrive in the work environment where, preferably, both clear expectations and instructions on their work tasks are specified (Parr, Hunter and Ligon, 2013). Finally, they prefer reasonable predictability in their work as they enjoy routines and if there is an unexpected change in their structured and precisely planned workday, they can sometimes get easily frustrated or even anxious (Cockayne, 2016; Hedley et al., 2018).

Interestingly though, some managers noted that an open communication style used by these employees had a positive impact on communication within the whole organisation. They also admitted that having these staff members on board made them better leaders (Hendrickx, 2008). Whereas other supervisors found their employees' straightforward communication style refreshing as, according to them, there was no "hidden agenda" (Hendrickx, 2008, p. 84). However, as reported by Hedley et al. (2018), this directness could cause tensions within a team and be sometimes taken by their colleagues for arrogance. For that reason, unfortunately, employees with AS are often misunderstood or not considered as team players (Pedersen, 2017). Transformational leadership, however, received an extensive critique for failing to promote inclusiveness within the organisation. As noted by Randel et al. (2017), reportedly, the leadership type known as inclusive was most suitable for employees with AS. Inclusive leaders embrace diversity and are able to utilise resources appropriately. This individualised consideration means that work tasks they assign match employees' predispositions (Parr, Hunter and Ligon, 2013). As a result, work performance improves whereas turnover rates decrease (Randel et al., 2017).

Scott et al. (2015) in their qualitative study contrasted viewpoints of 40 employees with AS with 35 employers which resulted in gathering a broader perspective on the topic of employment within this population. According to Scott at al. (2015), within organisations which truly promoted an inclusive culture, employers recognised employees' input and considered their viewpoints in their decision-making process. This meant employees felt appreciated and that their efforts were recognised which resulted in a noticeable increase in their intrinsic motivation. This made them more productive and engaged which in turn, benefited the whole organisation resulting in increased profits etc. Correspondingly, Randel et al. (2017) claimed that a leadership style classified as authentic also promoted inclusive culture but emphasised leader's ethical conduct. It was suggested that authentic leadership style is highly beneficial for employees with AS as they value qualities like honesty, integrity, respect, consideration for others, or fairness. Thus, if their leaders shared similar core values, 
they would experience greater job satisfaction and less anxiety (Parr, Hunter and Ligon, 2013).

The primary goal of this qualitative research was to explore the personal experiences of employees with AS in the workplace, their perceptions of workplaces and the challenges, if any, that they face at work. Furthermore, the present study aimed to explore hurdles related to the interview process and then the job retention, their work motivations, social interactions with colleagues, and their perceptions about the possible benefits of hiring employees with AS. Workplace improvements such as needs for reasonable adjustments, if required, and suggestions, including modifications in the hiring process to enable AS candidates to secure employment were also explored. As discussed by Krieger et al. (2012), there is a gap in research on the adult employees with AS, therefore, the current study provides a valuable contribution to the existing literature on this topic within the Irish context. Whereas, as emphasised by Hendrickx (2008), the stigma associated with AS is alive and well, including prejudice, misunderstandings and insufficient education on the topic within the general public. Thus, the current study has an educational value by addressing the existing stigma.

\section{Methodology}

\section{Participants}

Six participants living and working in Ireland, four females and two males, ranged in age from 27 to 50 (mean $=39.67$ years, SD $=8.16$ ), with a diagnosis of Asperger's Syndrome (AS) participated in this study. The inclusion criterion was any individual aged over 18, who self-identified as having AS, with participants recruited using a snowball and purposive sampling. Ethical approval for this study was granted by Dublin Business School Research Ethics Committee, and participant-informed consents were obtained prior to commencement.

\section{Design}

The interview topic guide was developed in order to explore job interview experiences, team interactions, physical environment and motivation at work. Ten open-ended questions were developed in cooperation with the second author and based on discussions and the previous literature. Semi-structured interviews were conducted with these questions to guide further discussion via a multimodal approach (three faceto-face, one by phone and two via Skype with the camera off). This method gives flexibility, allows for a natural conversation flow with a researcher open to anything that might come up in responses. It is also less intimidating to participants, enabling them to answer spontaneously whereas a researcher can gather more in-depth data (Pietkiewicz and Smith, 2014; Trevisan et al., 2017).

Qualitative interviews were digitally recorded and transcribed verbatim. The interviews were then thematically analysed to fully explore and summarise the employment experience of participants with AS (Braun and Clarke, 2006). The thematic analysis process comprised of data familiarisation through transcription and re-reading transcripts; line-by-line coding of each transcript and generation of a coding framework; creation of themes and sub-themes through synthesis of initial codes. A 
review of themes and sub-themes for appropriateness was conducted initially by the first author and subsequently in conjunction with the second author. This process allowed for theme interpretations to be validated through discussions and allowed for repeated referral to the coded extracts and original dataset; labelling and defining themes, the final step being the production of a written report of the analysis.

\section{Results}

Interviews lasted between 35 to 90 minutes, with an average duration of 62.5 minutes. Four main themes related to work experience of employees with AS emerged. These themes were: (1) Competence and Work Performance; (2) Self-improvement and Career Progression; (3) Supportive Work Environment; and (4) AS in the Workplace. An illustration of the themes and sub-themes is displayed in Figure 1. 


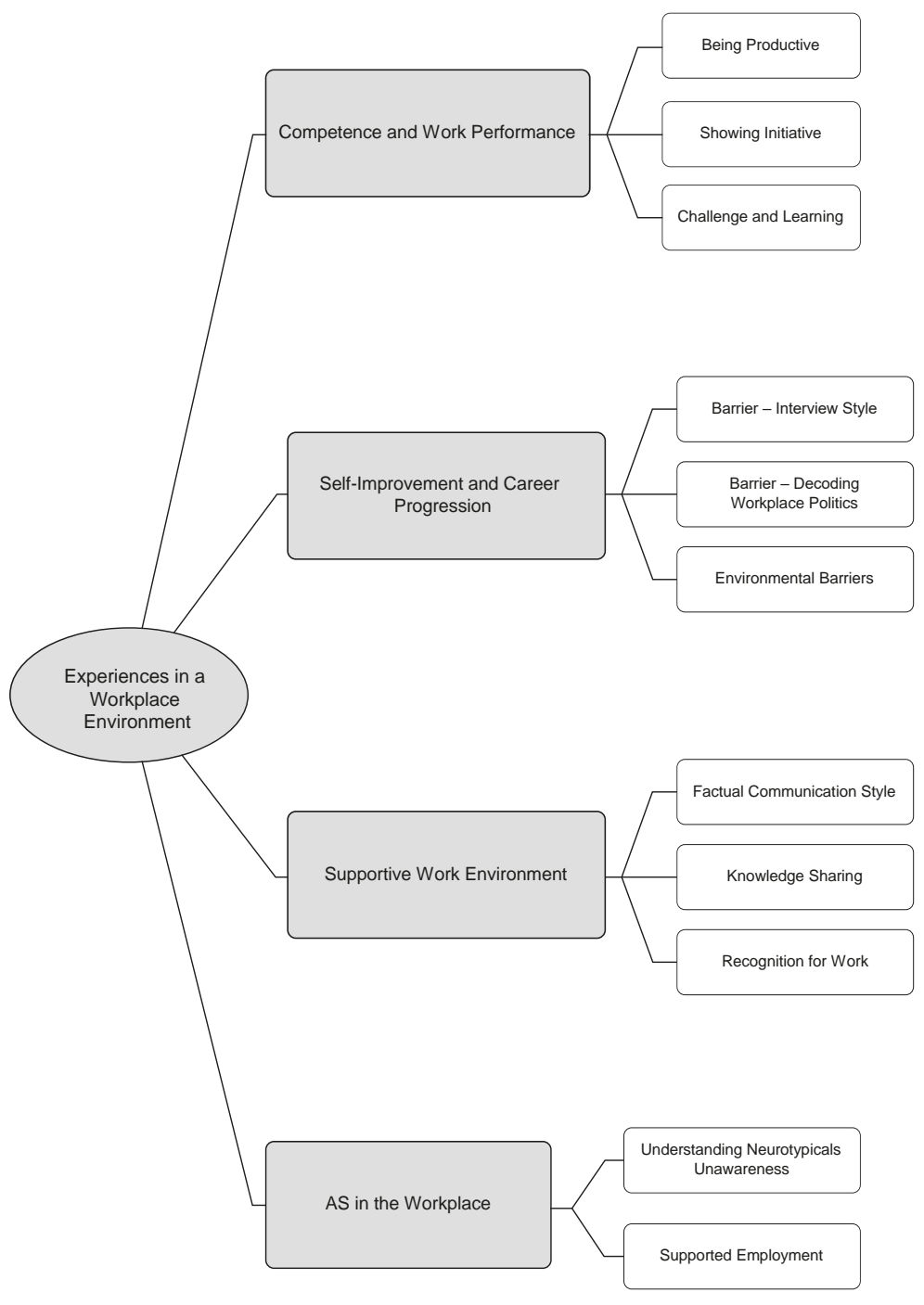

Figure 1. Thematic Map, illustrating four main themes (1) Competence and Work Performance; (2) Self-improvement and Career Progression; (3) Supportive Work Environment; and (4) AS in the Workplace and sub-themes.

\section{Competence and Work Performance}

Within this theme, the following three sub-themes emerged: (A) Being Productive; $(B)$ Showing Initiative; (C) Challenge and Learning. Most AS employees discussed how they feel confident about their skills. They identified themselves as highly-competent workers with an impeccable reputation and this confidence clearly showed in their responses.

Compared to my previous job, (in) this job I know they think I am doing a good job (...) so, when I tell him (the manager) that I have Asperger's, it is kind of like: 'That's great, well, we hear you are doing a great job'. (Participant B) 
Also, some admitted they took on a workload which normally would require additional resources. As reported by one participant, they believed they were more efficient than their colleagues. There was also a sense of achievement expressed by several participants, as they met the KPls and felt proud of having successful careers despite their diagnoses. They are often highly qualified and their strong sense of selfawareness about their advantages combined with a can-do attitude results in greater productivity.

Being productive was mentioned frequently, indicating that it means a lot to the participants. Not only do they prefer to be productive but also to report their actual productivity. As one participant outlined how their problem-solving skills contributed to greater efficiency at work. There was a strong willingness to be given sufficient workload and some reported how satisfying and motivating it was to work within a productive environment. It is also evident that they prefer to be occupied throughout their workday and use the time spent at work effectively as passively waiting for the tasks to be assigned frustrated them.

(...) my brain is always trying to think of things logically, you know, and always trying to do it the most effective and efficient way, it also means that I get tasks done faster than other people. (Participant C)

Some participants mentioned that they created their own work and responses revealed that apart from being highly productive, employees with AS showed a lot of initiative at work, for example creating files which were useful for the whole team. Interestingly, they were remarkably observant to quickly identify what was needed and then took full responsibility for tasks completion. Furthermore, they often performed tasks they were naturally good at, and, as a bonus, they also enjoyed performing them, saving their colleagues from engaging in tasks they probably found tedious. As a result, the resources were utilised adequately. Also, their ability to recognise what was necessary benefited the whole team. It means tasks were completed and anything overlooked by others was promptly identified and effectively resolved. They often applied problemsolving strategies, in this case, the solution was just to execute what was required.

I was thinking: 'We don't have team stats', so I started to create an Excel sheet, calculated team stats (...) I even wrote a handbook for beginners, when they are starting, with the training guide for the first five to six weeks because we never got anything like this. (Participant A)

Challenge and a quest for learning were mentioned frequently, particularly in the context of seeking challenge at work or not feeling challenged enough. There was a strong desire to work within an inspiring environment which gives sufficient opportunities to apply the skills they already had. Also, responses clearly indicated that to some, it was extremely motivating to learn at work every day.

I want the challenge every day, I want the challenge every minute (...) I'll do it, I'm able to work on some complex, whatever, but the thing is, where l'm now (...) there's no challenge because I can do everything. (Participant F) 
Moreover, to maximise quiet periods at work, self-training by means of work-related tutorials was applied by one of the participants rather than visiting entertainment sites on the internet and feeling unproductive. Therefore, responses clearly indicated that to some, it was essential to be given sufficient challenges, opportunities to continuously up-skill and trusting in their abilities to complete more complex tasks.

But just sitting down all the time and I feel worthless when I go home, and I haven't done anything (...) it is really terrible. I started learning, I was going through a lot of online trainings. (Participant $A$ )

\section{Self-improvement and Career Progression}

Three sub-themes were identified: (A) Barrier - Interview Style; (B) Barrier - Decoding Workplace Politics; (C) Environmental Barriers. This theme illustrates a strong willingness shared by participants to better themselves both on career and personal levels. Most of them mentioned how challenging it was at times for them to be in the workforce, however, they did not intend to use their diagnoses as excuses. In fact, they admitted how hard they have been working on themselves to combat obstacles caused by some of the symptoms.

So, what I did was, I started identifying those symptoms one at a time and I tackled them. (...) by necessity, the situation forced me to overcome those symptoms as well, which was a good thing. (Participant E)

Typical areas of difficulties like communication or single-tasking preference were addressed by making an extra effort i.e. learning social skills or doing their best to switch between tasks. One of the participants recognised the importance of communication within a team and they reported assigning a certain amount of time during the workday to socialise with work colleagues. The other wished to apply their learned social skills while participating in regular team meetings.

That's where I learned then, step by step, to focus on socialising skills. (...) It is just about $10-15 \%$ of my day that I only use for socialising then I know, at least 5 minutes per day to talk with everyone, so everyone is happy. And then, 'leave me alone'. (Participant A)

It was found that they were highly self-aware, recognised their weaknesses and stepped outside their comfort zones to tackle these limitations. As if overcoming difficulties was another problem to be solved, they tended to apply their problemsolving approach also in this case. Despite continuous self-improvement, the first obstacle to successful employment occurred at the recruitment stage. One participant replied that they found the note-taking person on the interview panel distracting and anxiety-provoking. Many participants critiqued the way job interviews were designed and some even believed that they were inappropriate for candidates with AS. Whereas others questioned the clarity of the competency-based interviews. The main areas of difficulties included literal interpretation of interview questions or audio distractions (background noise).

Like if I did a one-on-one interview that was taped and then they took notes afterwards, (it) would be easier than one person taking notes on the side... they are not asking any questions. (Participant B) 
This sub-theme addresses issues mentioned quite frequently by participants in relation to their inability to play workplace politics or understand team dynamics. As shared by some participants, it was mentally-taxing to them to decode these, almost as if it was a foreign language they could not comprehend. At the same time, this inability to either play or understand others playing politics was, as shown in responses, a massive barrier to promotion or maintaining employment.

I am usually the first one losing (in) this kind of games. I don't have the kind of skillset, or toolset for playing these games (...) it takes, at least $50 \%$ of my energy per day to just understand the social structure within this team. (Participant A)

Decoding team dynamics was closely related to difficulties in reading social cues, knowing when to join in conversations and responding adequately. Therefore, responses showed that manoeuvring within the world of social interactions was challenging and frustrating at times. Two core difficulties in the physical work environment appeared in most responses: the brightness of fluorescent lights and the noise, both being major sources of discomfort.

(...) I had to turn off the lights in my office because I couldn't function, it was too bright for me. (Participant D)

Another source of distress was noise and two participants reported that they would rather not go on their lunch breaks to avoid this over-stimulating environment. They found it difficult to filter out the noise and their way of dealing with it was to either take breaks when it was quiet or find an alternative place to go to. Participants often applied their problem-solving skills, for example they tried to switch these lights off whenever they could. Whereas the most popular solution to office noise was to use headphones or earplugs as sometimes it was the only way to work efficiently.

\section{Supportive Work Environment}

Three sub-themes were identified under this theme: (A) Factual Communication Style; (B) Knowledge Sharing; (C) Recognition for Work. In spite of some participants having a history of dealing with unsupportive former bosses, overall, they reported working currently in a reasonably supportive environment. Although some encountered bosses who were unwilling to make adjustments, even after a diagnosis disclosure, one participant's current manager understood the sensory overload difficulties allowing this employee to have a short break outside every time they felt overwhelmed.

(...) my boss is considerate, and, like, he tries to understand me and so, me and him, have, it's kind of like (...) we have a bond where we work well together. (Participant F)

Many enjoyed the fact that there was no micromanagement and they were given some degree of independence in their work. Others mentioned how much it meant to them for their manager to be open to inventive ideas and consider their suggestions. There was also no miscommunication as they often suggested having brief meetings with their managers to clarify any issues and receive feedback. Interestingly, they preferred their managers to be upfront. For employees with AS, managers' ability to listen, their honesty and openness to innovative ideas, combined with a willingness to assist with any work-related issues were essential. 
(...) in other jobs, l'd feel anxious all the time that I am doing something wrong or that $\mathrm{I}$, you know, there's an issue with something but they are being polite about it and they are not telling me. (Participant $\mathrm{C}$ )

Apart from directness, they prioritise factual communication and prefer to keep the small talk to an absolute minimum, focussing on discussing work-related matters when consulting their work. As per responses, communication, ideally, should be direct and work-focussed with professional interactions among team members indicating that these employees struggle with the unnecessary, in their opinion, conversation fillers. The fact that they wish to focus on work-related discussions explains why they are often very productive and have a strong work ethic, as discussed earlier.

(...) Just being really uncomplicated getting (a) quick question to a colleague, without any personal ego issues in the middle. (Participant A)

Knowledge sharing between different departments was equally important, but from one participant's point of view, unfortunately, a silo mentality was prevalent. It is also evident that asking work-related questions means a lot to them, as discussed by several participants. However, as observed by one participant, this may not always be welcomed by their colleagues as these questions were often highly-detailed. In their responses, participants expressed the importance of knowledge sharing within a team. Although working from home was distraction-free, one participant admitted they genuinely enjoyed being in the office as it gave them an opportunity to exchange ideas with others.

(...) they let me sit in on other department's meetings, but it was still in the technical department and, so then I could ask questions and then I knew that the part that I was doing was helping develop the frontend for this person's backend. You know, and it was just very enjoyable. (Participant $\mathrm{C}$ )

Seeking recognition was a recurrent pattern identified in many responses. Most participants reported that their managers recognised their good work, by, for example, acknowledging their organisational skills or initiative. However, some of them said that they did not always feel their effort was appreciated. One of the participants admitted that difficulties in decoding social cues could have resulted in them overlooking the appreciation expressed by their superiors. In some cases, participants either decided to move jobs when they felt underappreciated or their work was only recognised when they returned to the previous workplace. The need for recognition was evident in responses; this was expressed mainly as a wish to be appreciated for their contribution to work and receiving acknowledgement and support from the manager after tasks completion.

My boss (...) she won't necessarily tell you, you were brilliant, but you'd very soon know if there was a problem. (Participant D)

I constantly look for validation of people, say: "Thanks very much for that" (...). (Participant F) 
This theme represents how participants recognised AS's symptoms in people they worked with. Two sub-themes: (A) Understanding Neurotypicals Unawareness; (B) Supported Employment were identified. Interestingly, they nearly downplayed the severity of their own symptoms, while they could easily spot issues others had. It was as if they have that ease of almost diagnosing others.

I am convinced my manager possibly, well, my old manager, possibly would have had Asperger's herself. (Participant B)

One participant described their experience of being diagnosed with Asperger's prior to the amalgamation of diagnoses into ASD and that they went through the phase of denial and did not want to be classified as autistic, which they perceived as much more severe than Asperger's. Others disclosed that they felt they had it easier in comparison to others, their symptoms were milder and potentially because they were more selfaware. They often responded they were doing fine, were good in hiding their symptoms and did not really need any special adaptations but acknowledged others who, in their opinion, needed more assistance.

(...) I find ways to cope with it anyway, so, but the thing is, it's barely noticeable nowadays because (...) the situation forced me to overcome those symptoms as well, which was a good thing. (...) So these days it's barely noticeable (...). (Participant E)

Their responses suggest that they are observant, empathetic and value their selfsufficiency, whereas self-awareness makes them more mindful of others around them and some made a clear distinction between autism and Asperger's. Employees with AS do not necessarily expect others to understand their difficulties. They often acknowledged the fact that their colleagues may not have sufficient knowledge of AS. Some also understood that others' reactions to their struggles came from not understanding the condition rather than from malice.

I can't really expect everyone (by me just saying) I am just saying: 'I'm autistic' or 'I have Asperger's, by the way' that they'll understand what it means. (Participant A)

They also acknowledged differences in reasoning between themselves and their colleagues and believed that to be better understood and to minimise miscommunication, it was also their responsibility to educate others on Asperger's. Some shared their positive experiences of availing supported employment while job searching. One participant, for example, highly recommended contacting specialist AS employment services for anyone on the spectrum who struggled with securing employment, while the other was in the middle of the recruitment process with a perspective of getting a job offer.

So, with (Identifier Removed), they were sort of, they were unbelievably helpful (...) I had been searching for work for three years, so, I think that anyone who is searching for work and who has been long-term unemployed but who actually is definitely looking, 
like, who is eager to get work, l'd say (Identifier Removed) are definitely the first, like, l'd definitely put them as the first port of call (...). (Participant E)

However, one participant was hesitant and a bit sceptical about receiving assistance from organisations specific to supporting employees with AS. They expressed their concerns about the salary levels and that if they ever moved jobs, such work experience on their CV would need to be explained to their subsequent employer. As a result, it could be stigmatising and enforces the diagnosis disclosure prior to an interview. Responses show that it was a choice and conscious decision of each and every individual on the spectrum whether to secure employment with the assistance of specialist AS employment services or seek jobs independently.

\section{Discussion}

Results of the current study revealed that employees with a diagnosis of Asperger's perceive themselves to be highly-competent and professional workers. They discussed having a can-do-attitude and effective problem-solving skills. Moreover, interviewees gave numerous work-related examples that support their perspective that they are productive workers with a strong quest for upskilling. As per the findings of the current study, we suggest that their evident problem-solving skills were then translated into increased efficiency and meeting the expected KPIs. The findings of the present study align Wareham and Sonne (2008) who found that employees with a diagnosis of AS outperformed their work colleagues. Whereas the current study's findings are in opposition to some degree of uncertainty expressed by Scott et al. (2015) proposing that, perhaps, the diagnosed employees were not as efficient. Participants reported that their current managers were reasonably supportive although some of them admitted they sought more recognition for their hard work and contribution. Some of them specifically said that their ideal manager should be receptive to innovative ideas. Both recognition for work and open-mindedness correspond with the inclusive culture concept, as suggested by Scott et al. (2015).

Participants reported that they did not fear work-related challenges (for example to take on more demanding work tasks) and although some reported they did not necessarily enjoy unexpected changes within their work environment, overall they all sought opportunities at work to step outside their comfort zone. Interviewees also admitted that they had been making a great effort in areas they believed needed improvement, for example their social skills and more effective communication with other team members. Nonetheless, despite making an effort, several participants admitted that they experienced difficulties in decoding social cues in their teams. These findings correspond with results by Vogeley et al. (2013), however, this study's findings additionally revealed that employees with AS found participation in workplace politics mentally draining, consequently impairing their work performance and potentially hindering career progression. That, perhaps, explains why they prefer workrelated interactions to be factual and believe knowledge sharing among team members is essential. Contrary to the study by Parr, Hunter and Ligon (2013), the current findings indicate that employees with AS are team players and through knowledge-sharing or seeking work-related advice, they effectively cooperate with their colleagues. This could also be interpreted as a means of communication with a team. 
Employees with AS reported a preference for honest feedback from their managers, some reported taking the initiative to regularly have one-to-one meetings with their managers, again, evidencing a willingness to communicate. Moreover, many participants emphasised that direct communication with their team could minimise misunderstandings and prevent miscommunication. Wareham and Sonne (2008) reported similar findings that direct communication resulted in better communication across the whole organisation. Therefore, the current study's findings suggest that employees with AS are considerate team members who are willing to use effective communication strategies with consideration to healthy dynamics within their whole team. However, their strong sense of competence and work ethic could sometimes create frictions within the team as these could be perceived by others as overconfidence. As a result, their productivity, self-improvement or initiative could be entirely misunderstood by their colleagues, similarly. Their factual communication style, as discussed by Hedley et al. (2018), can be perceived as arrogance. An interesting finding of the present study was that participants described being proactive and self-sufficient at work and shared that they genuinely neither wanted major accommodations nor expected everyone to understand some of the difficulties they experienced at work. Perhaps, for that reason, they worked twice as hard on selfdevelopment.

Some of the recurrent themes found in participants' responses touched upon hurdles to either career-progression, or self-improvement, which included their inability to play and decode workplace politics. Whereas traditional interviews' styles were one of the barriers mentioned most frequently by participants, as these interviews were often a major source of distress, frustration or anxiety. As mentioned by Hendrickx (2008), apart from the understandable unwillingness or simply, fear of diagnosis disclosure at the interview stage, employees with AS who participated in the current study believed that job interviews they attended were not AS-friendly. A similar critique of the traditional interview process was mentioned by Austin and Pisano (2017).

Findings of the current study also revealed that environmental factors such as noisy surroundings like canteens and bright fluorescent lights were problematic to most employees with AS. Similarly, these environmental barriers were also highlighted by Parr and Hunter (2014) and Hedley et al. (2018). Although participants were resourceful and applied their problem-solving skills to alleviate these issues by either switching the lights off or avoiding the canteen during peak times at lunchtime, their responses showed that an over-stimulating environment compromised their well-being and comfort. Consequently, these findings suggest that decreased job satisfaction or considering the option of supported employment may occur for employees with AS.

Interestingly, despite some barriers or difficulties experienced at work, employees with AS nearly downplayed their symptoms whereas they easily identified AS traits in others. Moreover, participants believed that the symptoms they experienced were not as severe in comparison to others on the autistic spectrum. Similarly to Kite, Gullifer and Tyson. (2013), in the current study these responses were interpreted as a way of distancing from autism and its negative connotations thereby protecting themselves from prejudices or being stereotyped by others as having special needs or a disability. This corresponds with the doubt one of the participants of the current study shared during their interview. The dilemma was whether to avail of the services offered by 
supported employment or search for jobs independently to prevent being stigmatised when applying for prospective jobs. However, other participants had a positive experience with AS specific employment assistance services, and in the case of some participants, it resulted in being hired by the organisation which advocates and values inclusive culture. Moreover, they described greater job satisfaction in comparison to participants who never used the assistance of AS employment assistance services to find employment. This study's results correspond with the strengths of AS employment assistance services, as outlined by Austin and Pisano (2017). However, the current study gathered both advantages and disadvantages of this type of employment and this provides additional and, perhaps, more balanced insight. Supported employment services therefore may be less suitable for those who are unsure whether they wish their diagnosis to be known. As explained by Lorenz and Heinitz (2014), the diagnosis of AS could be stigmatising and detrimental to the self-worth of an employee, but the current study's findings did not find evidence to support it. In fact, this study's findings indicate that employees with AS described having stable self-esteem and believed their diagnoses were not obstacles to successful careers. Similarly to Krieger et al. (2012), participants of the current study emphasized how proud they were of their work accomplishments.

The current study is a valuable contribution to the existing research in workplace integration, through the exploration of experiences of adults with a diagnosis of AS, which may help to address the dearth of literature in this area and specifically within an Irish workplace context. The use of semi-structured interviews which resembled natural conversations, gave participants space and opportunity to share their workplace experiences (Vogeley et al., 2013; Pietkiewicz and Smith, 2014). As a result, the data gathered is rich in content. As already noted by Vogeley et al. (2013), previous studies narrowed their focus to the population of diagnosed children or their parents. Since autistic boys have been researched the most, this study has another asset: $66.7 \%$ of the sample were females. Up until now, studies including adult women with a diagnosis of AS have also been sparse. It needs to be considered that girls with a diagnosis of AS become women, but they do not grow out of AS. Therefore, we suggest that future research should include more female adults due to the existing gap in the literature. Another suggestion for future research is to give participants the option of giving written responses to interview questions. This would allow including the insights of participants who may find face-to-face communication with a stranger (i.e. a researcher) intimidating or anxiety-provoking (Griffith et al., 2012). It was recommended by Benford and Standen (2011) that this method of data collection is less energy- and mentally-taxing to some individuals with AS.

It is, however, worthwhile to note limitations of the current study, for example using the snowball sampling method which is prone to sampling bias. Its findings, though, could be limited to specific employees' population as $50 \%$ of them had a background in computer science. Moreover, either the age or gender of the researcher could have had an impact on participants-researcher interactions during interviews. As noted by Pietkiewicz and Smith (2014), female participants might have replied differently if a researcher was male. Since all participants extensively researched the topic of AS, the findings of this study represent their self-perceived beliefs and thus, should be interpreted with caution. 
Additionally, as mentioned by Hedley et al. (2018), it might be worth conducting more studies that would also focus on the population of managers and work colleagues of employees with a diagnosis of AS which may allow exploring both sides of the story and balancing out currently available research on the topic of employment and Asperger's. The current findings could be utilised when designing the workplace environment and may be a valuable source of information when developing training workshops to educate about creating an inclusive workplace environment. Such training may be a beneficial preventative measure used for stigma and existing stereotypes reduction. Moreover, such training may minimise misunderstandings, prevent conflict escalations caused by different communication styles or simply, as noted by Randel et al. (2017), reduce staff turnover. As a result, perhaps to some staff members with a diagnosis of AS, these workshops, by encouraging an open discussion on Asperger's, could bring relief and alleviate the apprehension to disclose their diagnosis.

The current study's findings could have some practical applications, particularly at the hiring stage. This emphasises the role of HR and how they could help candidates with a diagnosis of AS to show their strengths at the interview. Based on these findings, it is suggested that the traditional interview style may need some modifications to better support AS candidates. Modifications refer to including more knowledge-based questions and emphasising the assessment of these candidates' practical skills (Austin and Pisano, 2017). Another idea, as proposed by one participant, is to audiorecord interviews and reduce the interview panel to just one interviewer. Large numbers of people on panel-based interviews was a major source of distraction to the interviewee.

Managers who supervise AS employees may consider utilising more their problemsolving skills, whereas to motivate AS employees, they should clearly communicate recognition for their efforts and hard work but at the same time, when necessary, give them honest feedback on areas that need improvement. Participants disclosed they valued honesty, work relationships based on mutual trust and clarity in communication, and preferred constructive critique. Moreover, a lack of feedback on their performance makes them feel unsettled. Furthermore, the findings of this study suggest that AS employees benefited from regular one-to-one meetings. In line with Parr, Hunter and Ligon (2013) and Randel et al. (2017) that employees with AS sought a structured workplace, with clearly described, and communicated work goals. Also, managers should be approachable and, preferably, advocates of supportive and inclusive culture (Scott et al., 2015). In accordance with Parr, Hunter and Ligon (2013), the authors of this study additionally suggest that such an empowering leadership style was found to be the most suitable for employees with AS. The current study's findings also suggest that these employees excel when they work in innovation-promoting work culture as they reported they sought sufficient work-related challenges. However, to maximise their potential and increase job satisfaction, environmental factors as previously discussed may need to be addressed first.

This study's findings revealed that the diagnosis of AS is irrelevant to having a fulfilling career and that its participants expressed the universal basic human need to be appreciated for their hard work. Thus, regardless of the diagnosis, it is only natural that workers seek recognition for their efforts. Employees with Asperger's who 
participated in this study described themselves as productive, professional, and ambitious problem-solvers. Nonetheless, as found by Hedley et al. (2018), their selfconfidence could be misread as arrogance. That is why, to minimise such misunderstandings and to improve overall communication in the whole organisation, the authors suggested that brief educational workshops raising awareness on AS should be organised for all staff members. Based on the previous research by Parr and Hunter (2014) and Vogeley et al. (2013) and this study's findings, the authors concluded that AS employees are resilient individuals and high-achievers who often have successful careers in spite of numerous obstacles to and within their employment.

\section{References}

American Psychiatric Association (2013) Diagnostic and statistical manual of mental disorders (DSM-5). $5^{\text {th }}$ edn. Arlington, VA: American Psychiatric Publishing.

Austin, R.D. and Pisano, G.P. (2017) 'Neurodiversity as a competitive advantage', Harvard Business Review, 95(3), pp. 96-103. Business Source Complete, EBSCOhost [Online]. (Accessed: 24 October 2018).

Benford, P. and Standen, P.J. (2011) 'The use of email-facilitated interviewing with higher functioning autistic people participating in a grounded theory study', International Journal of Social Research Methodology, 14(5), pp. 353-368.

Braun, V. and Clarke, V. (2006) 'Using thematic analysis in psychology', Qualitative research in psychology, 3(2), pp. 77-101. Academic Search Complete, EBSCOhost [Online]. (Accessed: 1 October 2018).

Cockayne, A. (2016) "Hidden' disability: an investigation into the characteristics of employees with Asperger's syndrome'. Conference Abstract, Department of HRM, Nottingham Business School. Available at:

http://irep.ntu.ac.uk/id/eprint/29875/1/PubSub7239 Cockayne.pdf (Accessed: 2 February 2019).

Department of Health (2018) Estimating prevalence of Autism Spectrum Disorders (ASD) in the Irish population: a review of data sources and epidemiological studies. Available at: https://www.gov.ie/en/publication/0cc791-reports-on-the-prevalence-of-autism-in-irelandand-a-review-of-the-s/?referrer=/wp-content/uploads/2018/12/asd-report-final-19112018-forpublication.pdf/ (Accessed: 24 January 2019).

Griffith, G.M., Totsika, V., Nash, S. and Hastings, R.P. (2012) "I just don't fit anywhere': support experiences and future support needs of individuals with Asperger syndrome in middle adulthood', Autism, 16(5), pp. 532-546. Available at:

https://vcurrtc.org/research/documents/pdf/GriffithTotsikaNashandHastings2011.pdf (Accessed: 3 October 2018).

Hedley, D., Cai, R., Uljarevic, M., Wilmot, M., Spoor, J.R., Richdale, A. and Dissanayake, C. (2018) 'Transition to work: perspectives from the autism spectrum', Autism, 22(5), pp. 528- 
541. Available at: https://www.autisminwork.com/wp-content/uploads/2018/03/Hedley-et-al2017.pdf. (Accessed: 3 December 2018).

Hendrickx, S. (2008) Asperger syndrome and employment: what people with Asperger Syndrome really really want. London and Philadelphia: Jessica Kingsley Publishers.

Johnson, T.D. and Joshi, A. (2016) 'Dark clouds or silver linings? A stigma threat perspective on the implications of an autism diagnosis for workplace well-being', Journal of Applied Psychology, 101(3), pp .430-449.

Kite, D.M., Gullifer, J. and Tyson, G.A. (2013) 'Views on the diagnostic labels of autism and Asperger's disorder and the proposed changes in the DSM', Journal of autism and developmental disorders, 43(7), pp.1692-1700.

Krieger, B., Kinebanian, A., Prodinger, B. and Heigl, F. (2012) 'Becoming a member of the work force: perceptions of adults with Asperger Syndrome', Work, 43(2), pp.141-157. Business Source Complete, EBSCOhost [Online]. (Accessed: 1 October 2018).

Lorenz, T. and Heinitz, K. (2014) 'Aspergers - different, not less: occupational strengths and job interests of individuals with Asperger's Syndrome', PloS ONE, 9(6), pp.1-8. Academic Search Complete, EBSCOhost [Online]. (Accessed: 2 October 2018).

Lyons, V. and Fitzgerald, M. (2007) 'Asperger (1906-1980) and Kanner (1894-1981), the two pioneers of autism', Journal of Autism and Developmental Disorders, 37(10), pp. $2022-$ 2023. Available at:

https://www.researchgate.net/profile/Michael Fitzgerald15/publication/5923226 Asperger 1 906-1980 and Kanner 1894-

1981 the two pioneers of ASD/links/543c44290cf24ef33b7622be.pdf (Accessed: 19 February 2019).

Parr, A.D. and Hunter, S.T. (2014) 'Enhancing work outcomes of employees with autism spectrum disorder through leadership: leadership for employees with autism spectrum disorder', Autism, 18(5), pp. 545-554. Available at: https://bit.ly/35KXx2o (Accessed: 11 November 2018).

Parr, A.D., Hunter, S.T. and Ligon, G.S. (2013) 'Questioning universal applicability of transformational leadership: Examining employees with autism spectrum disorder', The Leadership Quarterly, 24(4), pp. 608-622. Available at: https://bit.ly/36TGAUT (Accessed: 11 November 2018).

Pedersen, K.N. (2017) Employees with Asperger syndrome - experiences and challenges. Master's thesis, UiT The Arctic University of Norway. Available at: https://munin.uit.no/bitstream/handle/10037/12591/thesis.pdf?sequence=1 (Accessed: 2 February 2019).

Pietkiewicz, I. and Smith, J.A. (2014) 'A practical guide to using interpretative phenomenological analysis in qualitative research psychology', Psychological Journal, 20(1), pp.7-14. 
Randel, A.E., Galvin, B.M., Shore, L.M., Ehrhart, K.H., Chung, B.G., Dean, M.A. and Kedharnath, U. (2017) 'Inclusive leadership: realizing positive outcomes through belongingness and being valued for uniqueness', Human Resource Management Review, 28(2), pp.190-203.

Scott, M., Falkmer, M., Girdler, S. and Falkmer, T. (2015) 'Viewpoints on factors for successful employment for adults with autism spectrum disorder', PloS ONE, 10(10), pp. 115. Academic Search Complete, EBSCOhost [Online]. (Accessed: 14 November 2018).

Skuse, D. (2018) 'Few people mourn Asperger syndrome's loss from diagnostic manuals'. Available at: https://www.spectrumnews.org/opinion/viewpoint/people-mourn-aspergersyndromes-loss-diagnostic-manuals/ (Accessed: 28 February 2019).

Trevisan, D.A., Roberts, N., Lin, C. and Birmingham, E. (2017) 'How do adults and teens with self-declared Autism Spectrum Disorder experience eye contact? A qualitative analysis of first-hand accounts', PloS ONE, 12(11), pp. 1-22. Academic Search Complete, EBSCOhost [Online]. (Accessed: 4 October 2018).

Vogeley, K., Kirchner, J.C., Gawronski, A., van Elst, L.T. and Dziobek, I. (2013) 'Toward the development of a supported employment program for individuals with high-functioning autism in Germany', European Archives of Psychiatry and Clinical Neuroscience, 263(2), pp.197-203. Academic Search Complete, EBSCOhost [Online]. (Accessed: 4 October 2018).

Wareham, J. and Sonne, T. (2008) 'Harnessing the power of autism spectrum disorder (Innovations case narrative: Specialisterne)', Innovations: Technology, Governance, Globalization, 3(1), pp.11-27. Available at:

https://www.mitpressjournals.org/doi/pdf/10.1162/itgg.2008.3.1.11 (Accessed: 29 November 2018). 